\title{
LA EVASIÓN TRIBUTARIA DEL IMPUESTO A LA RENTA EN LAS MYPES DEL RUBRO FERRETERİAS DE LA PROVINCIA DE SULLANA, PIURA
}

\author{
THE TAX EVASION OF INCOME TAX IN THE MYPES \\ DEL RUBRO FERRETERIAS OF THE PROVINCE OF SULLANA, PIURA
}

Adolfo Antenor Jurado Rosas?

\begin{abstract}
Resumen
Esta investigación tiene como objetivo determinar y describir la evasión tributaria del impuesto a la renta en las MYPES del Rubro Ferreterías de la provincia de Sullana, en Piura, a partir del alto índice de evasión tributaria que se evidencia en el pais y de las distintas modalidades que existen para llevar a cabo estas acciones ilícitas. Su carácter es descriptivo, con un enfoque cuantitativo de corte transversal; el tipo de investigación fue no experimental puesto que se realizó sin manipular deliberadamente las variables; así se observó el fenómeno tal como se encuentra dentro de su contexto. Como resultado se identificó que en lo referente a la evasión tributaria con una alta tasa de informalidad un $25 \%$ la realiza, mientras que un 75 \% no la realiza. La razón es porque tienen conocimiento de las consecuencias que puede ocasionar, por ello se recomienda que se tome la orientación correspondiente así no se infringe las leyes tributarias. Se llegó a concluir que las empresas no se acogen aún a la emisión de comprobantes electrónicos, ya sea por la falta de información o por la incapacidad de poder realizarlo puesto que este cambio influye mucho en el trabajo de la empresa, ya que se puede llegar a realizar una inversión para la correcta implementación de la facturación electrónica.
\end{abstract}

\section{Palabras clave}

Código tributario, evasión tributaria, impuesto a la renta, obligación tributaria, patrimonio neto, rubro ferreterías.

\begin{abstract}
The objective of this research is to determine and describe the tax evasion of income tax in the MYPES of the Hardware Stores of the province of Sullana from the high rate of tax evasion that is evidenced in the country and the different modalities that exist to carry carry out these illicit actions. The present research work was descriptive in nature, with a cross-sectional quantitative approach, the type of research was non-experimental because it was carried out without deliberately manipulating the variables, the phenomenon was observed as it is within its context. Obtaining as results the identification that within the tax doctrine referring to tax evasion with a high rate of informality, $25 \%$ do so, while $75 \%$ do not. Since they are aware of the consequences it can cause, it is therefore recommended that the corresponding guidance be taken so that tax laws are not violated. Coming to the conclusion that companies do not yet accept the issuance of electronic vouchers, either due to the lack of information or the inability to do so since this change greatly influences the work of the company, since it can be make an investment for the correct implementation of electronic invoicing.
\end{abstract}

Keywords

Tax Code, Tax Evasion, Income Tax, Tax Obligation, Net Worth, Hardware Stores.

JEL: E6-E63 


\section{Introducción}

La evasión tributaria conlleva una elevada controversia, debido a la mención de conductas ilícitas, la mayoría de los empresarios no lleva un registro de su evasión, y son las autoridades las que tienen la tarea de descubrir al evasor y el monto evadido, al cual se aplica después una sanción. Cuando las autoridades han descubierto a un evasor, este siempre recurre a argumentar que se trata de errores involuntarios por falta de comprensión de las normas, producto de su complejidad, malas recomendaciones de sus asesores tributarios, entre otros (Yañez, 2016)En cuanto a la realidad mundial Yáñez (2016) menciona que en varios de los países de América Latina, la evasión tributaria es una variante que toma relevancia en la explicación de la brecha tributaria, es decir, la diferencia entre lo que el gobierno debería recaudar, de acuerdo a lo planeado y el espíritu de la legislación tributaria y lo que realmente recauda.

Según la Comisión Económica para América Latina y el Caribe (Cepal, 2016), la evasión tributaria es uno de los principales puntos débiles de las economías de América Latina. La Cepal estima que en año 2015 la evasión ascendió a un monto equivalente a 2.4 puntos porcentuales del producto interno bruto (PIB) regional en el caso del impuesto al valor agregado (IVA) y 4.3 puntos del PIB en el caso del impuesto sobre la renta, lo que suma 340000 millones de dólares (6.7 \% del PIB en total).

La informalidad a nivel nacional se remonta muchos años, acentuándose con la presencia del comercio ambulatorio, los cuales son negocios que no se encuentran registrados ante la Superintendencia $\mathrm{Na}$ cional de Aduanas y de Administración Tributaria (SUNAT), tal y como lo establece la ley siendo un requisito de formalidad para ser controlados y supervisados como suje- tos pasivos de la relación jurídica tributaria (Choy, 2011, p. 2).

Pinedo y Delgado (2015) indican que la evasión tributaria incide negativamente en la liquidez y rentabilidad de las MYPE del Sector Comercial, debido a las multas impuestas por SUNAT, pero esta a su vez emplea estrategias deficientes en cuanto a la promoción de la cultura tributaria hacia los contribuyentes.

Yactayo (2016) hace mención a que SUNAT en los últimos tiempos no ha avanzado en cuanto a la reducción de la evasión tributaria, lo que deja en claro la falta de estrategias y de planes de acción para afrontar este problema. Complementando con la investigación de Yupa (2018) quien llega a determinar que la evasión tributaria influye negativamente en la recaudación de los tributos en las MYPE del sector comercio.

Ancco (2016) recomienda aplicar estrategias para incentivar la cultura tributaria en los contribuyentes otorgándoles incentivos tributarios por cumplimiento, incluso desde los programas educativos (Escuelas, Colegios y Universidades), con el fin de diseminar este problema de la sociedad civil.

\section{Antecedentes}

En cuanto a la realidad de Ecuador, Núñez (2015), propone identificar los determinantes para detectar las causantes de la evasión tributaria y los efectos en las finanzas de contribuyentes si aplican la normas tributarias adecuadamente, llegando a concluir que los contribuyentes no determinan el estado situacional, debido a que su operación constituye un fuerte impulso a la creación de empleo y aumento de ingresos de amplios segmentos de la población, esto representa un gran soporte en el desarrollo económico de la comunidad.

Pailán (2016) expone la realidad chilena teniendo como propósito caracterizar 
y comprender el sistema de antielusión tributaria en Chile, llegando a concluir que es posible observar el impacto que alcanza como consecuencia el fallo embonor en la comprensión de las normas tributarias, recalcando en ciertos criterios dados por los tribunales superiores que reconocieron los recursos representados por los contribuyentes, lo que indica que estos no comprenden la lógica de las normativas que rigen los tributos en el país, por lo cual incurren en errores tributarios y evasión.

En cuanto al caso colombiano, Heredia (2016) propone medir la presión fiscal sobre las Pyme colombianas en relación con el impuesto sobre la renta, teniendo como base la información del período de 20082012, llegando a concluir que en Colombia los términos de impuesto sobre sociedades e impuesto sobre la renta no tienen distinción, sino solo conocimiento de que son aplicables a personas naturales y personas jurídicas, respectivamente, logrando así un promedio de recaudación muy por debajo de los países como Argentina o Brasil.

En cuanto a la realidad nacional, Yactayo (2016) estableció si la evasión tributaria impuesto a la renta de tercera categoría de las MYPE tiene incidencia en la recaudación, tomando como punto crítico la evasión, debido a que es una práctica que impide la adecuada recaudación por parte del Estado; así llega a concluir que a pesar del gran trabajo que desempeña SUNAT el avance en cuanto a reducción de la evasión tributaria es muy poco, lo que evidencia la aplicación de malas estrategias en la difusión de la cultura tributaria.

Yupa (2018) propone como meta determinar la repercusión de la evasión tributaria del impuesto a la renta de tercera categoría en la recaudación tributaria de las MYPE, llegando a concluir que la evasión repercute en la recaudación tributaria, esta situación incide en el estudio de Roca (2016) quien propuso investigar sobre la evasión tributaria del IGV y su efecto en la recaudación tributaria, a lo que concluye que los empresarios se rehúsan a cumplir con las obligaciones tributarias, debido a la poca información brindada o deficiente asesoría por parte de los contadores o la misma SUNAT.

En cuanto al ámbito local, Marino (2016) que propone analizar los factores causantes de la informalidad y la evasión tributaria de las MYPE, concluye que la evasión tributaria está estrechamente relacionada con la informalidad de los empresarios debido al desconocimiento de las normativas que regulan la recaudación tributaria, desconocimiento de los beneficios de la formalidad, ineficiencia de la gestión realizada por SUNAT y la falta de conciencia tributaria.

Mena (2019) recalca que la aplicación de un adecuado planeamiento tributario, reduciría la evasión presentada en las empresas, pero los empresarios no están dispuestos a aplicarlo por la falta de normativas tributarias, incurriendo en la evasión.

\section{Marco teórico}

El sistema tributario peruano se compone de principios, reglas y normas las cuales han establecido los tributos, desenvolviéndose dentro del marco conceptual que le brinda el Derecho Tributario y la Ley Penal Tributaria. Este sistema se caracteriza por ser suficiente y elástico, el Estado combina todos los impuestos dentro de este sistema; lo recaudado es destinado para el Tesoro Público, quien provee en el Presupuesto General, de esta manera el Estado tiene un "colchón financiero" y no incurre en la creación de nuevos impuestos (SUNAT, 2017).

Al ser de derecho público la obligación tributaria constituye el vínculo entre el acreedor y el deudor tributario, según lo establecido por ley tiene como propósito el cumplimiento de la prestación tributaria y tiene exigencia 
coactiva. Se dividen en obligaciones formales (en las cuales el contribuyente cumple con el otorgamiento de los comprobantes de pago por las ventas realizadas, presentación de declaraciones juradas y otras comunicaciones, el llevado de libros y registros contables) y obligaciones sustanciales destinadas a la determinación y pago de los tributos que corresponden a cada contribuyente (Pinedo y Delgado, 2015).

Esto nace cuando se realiza el hecho previsto en la ley, como generador de dicha obligación (Actualidad Tributaria, 2018), en esa instancia la obligación tributaria es exigible cuando deba ser determinada por el deudor tributario desde el día siguiente al vencimiento del plazo ya fijado por la Ley de acuerdo al artículo 29 de este código y cuando es determinada por la SUNAT desde el día siguiente después del vencimiento. (Actualidad Tributaria, 2018).

En caso la obligación sustancia y/o formal es incumplida, nace la infracción tributaria la cual se diferencia de un delito porque no ataca directamente los derechos de los ciudadanos, tiene un carácter contravencional por ser una obstrucción ilícita a la formación de rentas públicas. (Flores, 2007, p. 203)

Existen dos tipos de sujetos de la infracción tributaria, el sujeto activo es aquel que realiza la acción tipificada como infracción sancionable y sobre quien recae la infracción en materia tributaria y el sujeto pasivo el cual es el titular del bien jurídico afectado por la infracción. (Flores, 2007, p. 203)

Las sanciones correspondientes se separan en dos grupos, el primero son las sanciones pecuniarias como las sanciones y multas, el segundo grupo son la no pecuniarias quienes incluyen el cierre de locales, comiso de bienes, cancelación de licencias e internamiento de vehículos. (PUCP, 2017)

En cuanto a la evasión tributaria es la disminución del monto tributario produ- cido dentro del país por parte de quienes están obligados a abonarlo y no lo hacer, en cambio obtienen beneficios mediante comportamientos fraudulentos, diferenciándose la evasión legal y la evasión ilegal". (Camargo, 2005, p. 25)

La elusión tributaria es aquella conducta del contribuyente el cual busca evitar el pago de impuestos utilizando maniobras o estrategias permitidas por los vacíos de la ley, esto indica que la elusión no es estrictamente ilegal ya que no hay violación de ley alguna, sino que es un aprovechamiento de los vacíos de esta. (Gerencia, 2018)

Otras de las modalidades de la elusión tributaria es darle a una situación jurídica determinada el estatus de otra distinta, aprovechando alguna clase de conceptualización defectuosa, para aplicarle una ley tributaria más benigna. Ampararse en una exención tributaria que, por defectuosa técnica legislativa, no había sido prevista para el caso particular en cuestión. Incluir bienes en categorías cuya enumeración no está contemplada por la ley tributaria, y por tanto su inclusión dentro del hecho tributario es dudosa, para eximirlos de impuestos. (Pinedo \& Delgado, 2015)

Esto tiene varias causas de acuerdo con Yacolta (2018) las cuales pueden ser: económicas, técnicas, políticas y jurídicas.

En cuanto al impuesto a la renta ya que este es un tributo que se calcula anualmente y se considera como un ejercicio gravable aquel que comienza el 1 de enero y termina el 31 de diciembre de cada año; grava las rentas que provengan de la explotación de un capital (bien mueble o inmueble), las que provengan del trabajo realizado en forma dependiente e independiente, las obtenidas de la aplicación conjunta de ambos factores (capital y trabajo), así como las ganancias de capital. (Morales, 2010)

Por todo lo mencionado, el objetivo de nuestro estudio ha sido determinar y describir la evasión tributaria del impuesto a la 
renta en las MYPES del Rubro Ferreterías de la provincia de Sullana a partir del alto índice de evasión tributaria que se evidencia en el país y las muchas modalidades que existen para llevar a cabo estas acciones ilícitas.

\section{Metodología}

\section{Diseño de investigación}

El presente trabajo de investigación fue de carácter descriptivo (Valderrama, 2013), con un enfoque cuantitativo de corte transversal, el tipo de investigación fue no experimental (Hernández, Fernández y Baptista, 2014) debido a que se realizó sin manipular el comportamiento de las variables, así se observó el fenómeno tal como se encuentra dentro de su contexto.

\section{Población y muestra}

Se tuvo una población compuesta de los propietarios de las empresas del rubro ferreterías de la ciudad de Sullana en el año 2019, según el Registro de Licencias de Funcionamiento se identificó a cuatro empresas sin aplicar una técnica de muestreo, esto debido a que la población es demasiada pequeña.

\section{Instrumento}

El instrumento que se utilizo fue el cuestionario compuesto por preguntas cerradas, las mismas que fueron aplicadas a propietarios de las empresas ferreteras, dicho instrumento fue validado por expertos teniendo un coeficiente de alfa de Cronbach de 0.802 la cual es una alta confiabilidad.

\section{Plan de análisis}

Para el tratamiento del acopio de información, se procedió a la tabulación utilizándose el programa IBM SPSS versión 25.0, en donde se detalló cada variable de estudio. La pre- sentación de resultados se realizó mediante tablas de frecuencia absoluta y gráficos.

\section{Consideraciones éticas}

En el trabajo de investigación, se respetó la confidencialidad ofrecida en el consentimiento informado, el manejo restringido de dicha data. Asimismo dicho trabajo fue aprobado por el Comité Institucional de Ética en Investigación de la Universidad Católica Los Ángeles de Chimbote (CIEI-ULADECH católica), quien emitió informe de autorización n. 010-2016-CEI-VI-ULADECH-católica.

\section{Resultados}

De un total de cuatro propietarios de las empresas correspondientes al sector comercio, se obtuvieron los siguientes resultados:

Se identificó con respecto a la emisión de facturas que el $25 \%$ de los encuestados tiene conocimiento acerca de la Ley de Bancarización, también se observa que el 100\% de los encuestados no realizan la emisión de facturas electrónicas, el mismo comportamiento se evidencia con la emisión de boletas electrónicas. Respecto a la elusión tributaria, se observa que en cuanto a la doctrina tributaria el $75 \%$ de los encuestados se encuentra con un alto porcentaje de informalidad.

En cuanto al análisis de la cultura tributaria, el $75 \%$ de los encuestados considera que las infracciones administrativas cometidas no están relacionadas con el desconocimiento y la capacitación tributaria, sino que el $75 \%$ considera que los impuestos no tienen una utilidad benéfica para la sociedad y es el mismo porcentaje que nunca ha recibido asesoramiento en cuanto a la presentación de declaraciones tributarias. El 75\% que la acción de ocultar bienes o ingresos de SUNAT para reducir el pago de los impuestos está más relacionado a un tema de estrategia y no por falta de cultura tributaria. 
Tabla 1. Características de la Evasión Tributaria

al Impuesto a la Renta de 4 empresas del Perú

\begin{tabular}{|c|c|c|c|c|}
\hline Dimensiones & Indicadores & Items & Respuesta & $\%$ \\
\hline \multirow{8}{*}{$\begin{array}{l}\text { Comprobante } \\
\text { de pago }\end{array}$} & \multirow{4}{*}{ Facturas } & \multirow{2}{*}{ Considera la Ley de Bancarización. } & $\mathrm{Si}$ & $25 \%$ \\
\hline & & & No & $75 \%$ \\
\hline & & \multirow{2}{*}{ Emisión de facturas electrónicas. } & Sí & $0 \%$ \\
\hline & & & No & $100 \%$ \\
\hline & \multirow{4}{*}{ Boletas } & \multirow{2}{*}{$\begin{array}{l}\text { Monto mínimo y máximo para emitir una } \\
\text { boleta. }\end{array}$} & Sí & $25 \%$ \\
\hline & & & No & $75 \%$ \\
\hline & & \multirow{2}{*}{ Emite boleta electrónicos. } & Sí & $0 \%$ \\
\hline & & & No & $100 \%$ \\
\hline \multirow{4}{*}{ Elusión Tributaria } & \multirow{2}{*}{$\begin{array}{l}\text { Doctrina } \\
\text { tributaria. }\end{array}$} & \multirow{2}{*}{ Evasión tributaria - Alta tasa de informalidad. } & Sí & $25 \%$ \\
\hline & & & No & $75 \%$ \\
\hline & \multirow{2}{*}{ Leyes tributarias. } & \multirow{2}{*}{$\begin{array}{l}\text { Evasión tributaria es un acto ilícito que afecta } \\
\text { al Fisco }\end{array}$} & Sí & $25 \%$ \\
\hline & & & No & $75 \%$ \\
\hline \multirow{8}{*}{ Cultura tributaria } & \multirow{2}{*}{$\begin{array}{l}\text { Aspectos } \\
\text { normativos }\end{array}$} & \multirow{2}{*}{$\begin{array}{l}\text { Infracciones administrativas son por carencia } \\
\text { de orientación y capacitación tributaria. }\end{array}$} & Sí & $25 \%$ \\
\hline & & & No & $75 \%$ \\
\hline & \multirow{4}{*}{$\begin{array}{l}\text { Aspectos } \\
\text { culturales }\end{array}$} & \multirow{2}{*}{ Utilidad de los impuestos. } & Sí & $25 \%$ \\
\hline & & & No & $75 \%$ \\
\hline & & \multirow{2}{*}{$\begin{array}{l}\text { Asesoramiento para las declaraciones } \\
\text { mensuales. }\end{array}$} & Sí & $25 \%$ \\
\hline & & & No & $75 \%$ \\
\hline & \multirow{2}{*}{$\begin{array}{l}\text { Aspectos } \\
\text { socio-económicos }\end{array}$} & \multirow{2}{*}{$\begin{array}{l}\text { Ocultar bienes o ingresos para pagar me- } \\
\text { nos impuestos se debe a la falta de cultura } \\
\text { tributaria. }\end{array}$} & Sí & $25 \%$ \\
\hline & & & No & $75 \%$ \\
\hline
\end{tabular}

Fuente: Elaboración propia.

\section{Discusión}

De acuerdo a los resultados se pudo identificar que, con respecto a la dimensión de los comprobantes de pagos, estos muestran que un $25 \%$ de los encuestados si consideran la Ley de Bancarización, mientras que el $75 \%$ refleja que no la toman en cuenta; también se identifica también, respecto a la emisión de facturas electrónicas, que un 100 \% de los encuestados no la realizan, lo mismo sucede respecto a la emisión de boletas de ventas electrónicas, puesto que un $100 \%$ no las realiza. Esto se debe a que aún no cumplen con las características para ingresar a la emisión de comprobantes de pago electrónicos, por ello se recomienda el que se puedan acoger a dicha afiliación.
En cuanto a la elusión tributaria se identificó que dentro de la doctrina tributaria referente a la evasión tributaria con una alta tasa de informalidad un 25\% lo realiza, mientras que un $75 \%$ no lo realiza. Esto se da porque tienen conocimiento de las consecuencias que puede ocasionar, por ello se recomienda que se tome la orientación correspondiente así no se infringen las leyes tributarias.

Todo lo señalado se relaciona con la investigación del autor Yáñez (2016) quien menciona que en los países de América Latina, la evasión tributaria es una variable que toma importancia en la explicación de la brecha tributaria, es decir la diferencia entre lo que el gobierno debería recaudar, de acuerdo a lo planeado y el espíritu de la legislación tributaria y lo que realmente recauda. 
En lo que se refiere a la cultura tributaria se refleja que en las infracciones administrativas que son por carencia de orientación y capacitación tributaria un 25\% lo realiza, mientras que un $75 \%$ no, y eso se debe al constante seguimiento que realiza SUNAT del pago de los impuestos correspondientes.

\section{Conclusión}

Se concluye que los empresarios aún no se acogen a la emisión de comprobantes electrónicos, ya sea por la falta de información o por la incapacidad de poder realizarla, puesto que este cambio representa en el trabajo de la empresa, una gran inversión para su correcta implementación de la facturación electrónica que garantice el cumplimiento tributario de forma automática con SUNAT.

Otra de las conclusiones importantes es que se pudo identificar que los empresarios tienen algún conocimiento de las infracciones administrativas puesto que SUNAT realiza un adecuado seguimiento en cuanto a las obligaciones que se deben cumplir. Además se observó que los mismos no se encuentran asesorados en materia tributaria para el cumplimiento de las declaraciones mensuales puesto que más de la mitad lo desconoce, ocasionando una insatisfacción con respecto a los pagos de impuestos.

\section{Referencias}

Actualidad Tributaria. (2018). Libro Primero La Obligación Tributaria Título I Disposiciones Generales-Art. $1^{\circ} 2^{\circ}$. ACTUALIDAD TRIBUTARIA, (p. A-9).

Actualidad Tributaria. (2018). Libro Primero La Obligación Tributaria Título I Disposiciones Generales-Art.3․ ACTUALIDAD TRIBUTARIA (p. A-9).

Ancco, C. (2016). Caracterización de la evasión tributaria del impuesto a la renta de tercera categoría caso MYPE Petshappy EIRL, Rubro Veterinaria y su Incidencia en la
Recaudación Tributaria Cercado de Lima, 2015. Lima - Perú. Recuperado el 28 de 03 de 2020

Camargo, D. (2005). Evasión fiscal un problema sin resolver. www.eumed.net/libros/2005/dfch-eva/

Cepal. (septiembre de 2016). Comisión Económica para América Latina y el Caribe. Recuperado de https://www.cepal.org/es/ noticias/evasion-fiscal-america-latina-llega-340000-millones-dolares-representa67-pib-regional

Choy, E. y Montes, E. (2011). La informalidad en los sectores económicos y la evasión tributaria en el Perú. QuipuKamayoc. Revista de la Facultad de Ciencias Contables, 18(35) Recuperado el 17 de 09 de 2019. https:// doi.org/10.15381/quipu.v18i35.6805

Echevarría, A.; Morales, J. R. y Varela L. (2006). Alternativas de financiamiento para las pequeñas y medianas empresas (PYMES) mexicanas. Revista académica de economía. Observatorio de la Economía Latinoamericana 80.

Flores, J. (2007). Auditoria Tributaria Procedimiento y Técnicas de AT Teoría y Práctica. CECOF Asesores E. I. R. L. (2. ${ }^{a}$ ed). Recuperado el 26 de 03 de 2020

Gerencia. (2018). Elusión y evasión tributaria. Recuperado de www.gerencia.com/elusion-y evasión - tributaria.html

Gómero, N. A. (2005). Participación de los intermediarios financieros bancarios y no bancarios en el desarrollo de las pymes textiles en Lima Metropolitana de 1990 al 2000. Lima - Perú. Recuperado de www. unsm.edu.pe/

Heredia, L. (2016). Presión fiscal sobre las PYMES: una aplicación al caso colombiano del impuesto sobre la renta. Tesis Doctoral, Universidad de Salamanca, Facultad de Derecho, Salamanca - España. Recuperado el 08 de 09 de 2020.

Hernández, R.; Fernández, C. y Baptista, M. (2014). Metodología de la investigación. McGraw-Hill. Recuperado el 28 de Enero de 2020, de file:///P:/LIBROS\%20DE\%20 TESIS/METODOLOGIA\%20DE\%20LA\%20 INVESTIGACION\%20-\%20SAMPIERI.pdf 
Hinojosa, C. (2012). Impacto de la Remuneración Mínima en el Desarrollo Socioeconómico de los Trabajadores de las Micro y Pequeñas Empresas de la Ciudad de Chachapoyas, año 2012. Chachapoyas - Perú.

Marino, M. (2016). Análisis de los factores causantes de la informalidad y evasión tributaria de las MYPE comercializadoras de ropa en Piura. Tesis para obtener el grado de magíster, Universidad Nacional de Piura, Escuela de Posgrado, Piura-Perú. Recuperado el 08 de septiembre de 2020.

Medina, L. y Flores, F. (2010). Incidencia del financiamiento y la capacitación en la rentabilidad de las MYPES del sector turismo de la localidad de Huancavelica año 2008.

Mena, M. (2019). La evasión tributaria y su incidencia en las micro y pequeñas empresas del sector comercio rubro restaurantes en el Perú Caso: Pollería Gisela E.I.R.L., ciudad de Piura 2019. Tesis para optar el título profesional de contador público, Universidad Católica Los Ángeles de Chimbote, Facultad de Ciencias Contables, Financieras y Administrativas, Piura-Perú. Recuperado el 08 de septiembre de 2020.

Morales, C.; Ruiz, F.; Ycaza; J. (2010). Análisis de la evasión fiscal en el impuesto a la renta del Ecuador.

Núñez, A. (2015). Determinantes económicos en la recaudación fiscal de las PYMES de la provincia de Santa Elena. Maestría en Economía con Mención en Finanzas y Proyectos Corporativos, Universidad de Guayaquil, Facultad de Ciencias Económicas, Guayaquil - Ecuador. Recuperado el 08 de septiembre de 2020. http://repositorio. ug.edu.ec/handle/redug/8967

Olano. (2012). Las MYPE y las Dificultades de Acceso a las Fuentes de Financiamiento.

Pailán, R. (2016). El sistema de antielusión tributaria en Chile. Tesis para optar el grado de magíster. Universidad de Chile, Economía y Negocios, Santiago de Chile - Chile. Recuperado el 08 de septiembre de 2020. epositorio.uchile.cl/bitstream/ handle/2250/144262/Paillán\%20An camil\%20Ramón.pdf?sequence $=1 \&$ is Allowed $=\mathrm{y}$
Pinedo, L., y Delgado, R. (2015). Incidencia de la evasión del impuesto a la renta en la situación económica y financiera de las MYPES del Sector Abarrotes en el Distrito de Tarapoto, período 2013. Tarapoto - Perú. Recuperado el 28 de 03 de 2020.

PUCP. (2017). Cual es la diferencias entre infracción y sanción tributaria. Obtenido de http://blog.pucp.edu.pe/item/187620/ cu-l-es-la-diferencia-entre-infracci-n-ysanci-n-tributaria.

Rengifo. J. (2010). Caracterización del financiamiento, la capacitación y la rentabilidad de las micro y pequeñas empresas del sector comercio-rubro artesanía shipibo - Conibo del distrito de Callería - provincia de Coronel Portillo, período 2009- 2010. Universidad Católica los Ángeles de Chimbote. Pucallpa.

Roca, Y. (2016). La evasión tributaria del IGV de las MYPES los Artesanos EIRL del rubro tapiceria y su efecto en la recaudación de impuestos en el Cercado de Lima, 2015. Tesis para optar el título profesional de contador público, Universidad Católica Los Ángeles de Chimbote, Facultad de Ciencias Contables, Financieras y Administrativas, Lima - Perú. Recuperado el 08 de septiembre de 2020. http://repositorio. uladech.edu.pe/handle/123456789/1745

SUNAT. (2017). Sistema Tributario. Recuperado el 28 de 03 de 2020, de http://www.sunat. gob.pe/institucional/quienessomos/sistematributario.html

Trujillo, M. (2010). Caracterización del financiamiento, la capacitación y la rentabilidad de las MYPES del Sector Industrial - Rubro Confecciones de Ropa Deportiva del Distrito de Cercado de la Provincia de Arequipa, Periodo 2008-2009. Arequipa - Perú.

Valderrama, S. (2013). Pasos para elaborar proyectos de investigación cientifica. San Marcos E.I.R.L.

Yacolta, D. (2018). Perú causas de evasión tributaria. Obtenido de www.sunat.gob.pe

Yactayo, G. (2016). Caracterización de la evasión tributaria del impuesto a la renta-tercera categoría de las MYPES rubro transporte, distrito de la Victoria - Lima 2015. Tesis 
para optar el título profesional de contador público, Universidad Católica Los Ángeles de Chimbote, Facultad de Ciencias Contables, Administrativas y Financieras, Lima - Perú. Recuperado el 08 de septiembre de 2020. http://repositorio.uladech. edu.pe/handle/123456789/1333

Yañez, J. (2016). Evasión tributaria: atentado a la equidad. Centro de Estudios Tributarios, 36. Recuperado el 17 de septiembre de 2019

Yupa, E. (2018). Caracterización de la evasión tributaria, impuesto a la renta de tercera categoría y su incidencia en la recaudación tributaria en las MYPES del Sector Comercio - Rubro Abarrotes del distrito de Juliaca, periodo 2017. Tesis para optar el título profesional de contador público, Universidad Católica los Ángeles de Chimbote, Facultad de Ciencias Contables, Financieras y Administrativas, Juliaca - Perú. Recuperado el 08 de septiembre de 2020. http://repositorio.uladech.edu.pe/handle/123456789/2934 